\title{
Standard orthotopic heart transplantation
}

\author{
Jérôme G. M. Jungschleger ${ }^{1}$, Sergey Yu Boldyrev ${ }^{2,3}$, Vasily I. Kaleda ${ }^{2}$, John H. Dark ${ }^{4}$ \\ ${ }^{1}$ Institute of Transplantation, Department of Cardiothoracic Surgery, Freeman Hospital, Newcastle upon Tyne, UK; ${ }^{2}$ Department of Adult Cardiac \\ Surgery, Ochapowski Regional Hospital \#1, Krasnodar, Russia; ${ }^{3}$ Division of Cardiac Surgery and Cardiology, Kuban State Medical University, \\ Krasnodar, Russia; ${ }^{4}$ Institute of Cellular Medicine, Faculty of Medical Sciences, Newcastle University, Newcastle upon Tyne, UK \\ Correspondence to: Professor John Dark. Institute of Cellular Medicine, Faculty of Medical Sciences, Newcastle University, Newcastle upon Tyne, UK. \\ Email: John.Dark@ncl.ac.uk.
}

Submitted Jan 13, 2018. Accepted for publication Jan 15, 2018.

doi: 10.21037/acs.2018.01.18

View this article at: http://dx.doi.org/10.21037/acs.2018.01.18

\section{Introduction}

The very first clinical transplants used a technique, with anastomoses of atrium to atrium, which had been developed to allow successful canine experiments (1). A small modification by Barnard led the suture line away from the sino-atrial node. Many years later Sievers (2) described the intact right atrium, with separate caval anastomoses, now the standard procedure, with a proven decrease in need for permanent pacemakers. A further refinement, with intact left atrium and separate pulmonary venous cuff anastomoses (3), the "total orthotopic transplant" has not proven popular.

\section{Clinical vignette}

The recipient was a 43-year-old male with a diagnosis of dilated cardiomyopathy associated with myocarditis. He was in heart failure (HF) NYHA class IV, pulmonary edema, inotropic support, equivalent to Status $1 \mathrm{~B}$. Left ventricular ejection fraction (LVEF) varied from $10 \%$ to $16 \%$; mitral regurgitation (MR) ++; tricuspid regurgitation (TR) +++; pulmonary artery pressure (PAP) $40-45 \mathrm{mmHg}$. Ventricular fibrillation developed during right heart catheterization. Defibrillation was successful, but the patient's condition deteriorated rapidly and required inotropic support. All the measures were performed under infusion of epinephrine $0.2 \mathrm{mg} / \mathrm{kg} / \mathrm{min}$ : CI $1.8 \mathrm{~L} / \mathrm{min} / \mathrm{m}^{2}$, PVR 1.4 WU, TPG $6 \mathrm{mmHg}$. The transplantation was performed in 2015. Histology of the explanted heart showed diffuse myocardial fibrosis. At follow-up the patient remains in a satisfactory condition 2 years after surgery.

\section{Technique and comments}

\section{Preparation}

After confirmation of a potentially suitable donor, the shaved, washed ( $4 \%$ chlorhexidine) and starved patient is taken to theatre. The timings should allow comfortable anaesthetic induction and recipient heart excision before arrival of the donor heart in order to keep ischaemic time as short as possible. Anaesthesia is not induced until the donor heart acceptability is confirmed. A trans-oesophageal echo probe and Swan-Ganz catheter positioned in the superior vena cava (SVC) is our standard monitoring beyond the routine.

\section{Operation}

\section{Recipient heart excision}

The chest is opened through a median sternotomy, followed by a vertical pericardiotomy. The aorta and pulmonary artery are completely separated and the cavae mobilised and tapes passed around both. After heparinization, the aortic cannula is inserted high in the ascending aorta. We cannulate the SVC with a right-angled cannula located just proximal to the tape snare, and well above the cavoatrial junction. A straight cannula is passed into the inferior vena cava (IVC) through a purse string just above the diaphragmatic reflection.

Cardiopulmonary bypass can now be established with mild hypothermia $\left(32{ }^{\circ} \mathrm{C}\right)$. When the donor heart is within 10-15 minutes of arriving, the caval vein snares are tightened and the aortic cross clamp positioned as distal 
as possible. Excision of the heart starts by incising the left atrium at the junction of the right superior pulmonary vein, which decompresses the left heart. This incision can be extended in each direction. The SVC is divided at the cavo-atrial junction. After incising the right atrium above the IVC cannula, to leave at least $1 \mathrm{~cm}$ of right atrial cuff above the tape snare, the right atrium is completely divided. The aorta and then pulmonary artery are transected just above the valves, to leave a good length of distal vessel. Finally, the incision in the left atrium is extended medially across the roof and down in front of the appendage. The atrial incisions are joined up, to leave the four pulmonary veins in situ as a large cuff, and the recipient heart excised. It is prudent to check for haemostasis around the posterior aspects of the pericardial cavity after cardiectomy.

\section{The donor heart}

If the lungs have not been retrieved, the first step is to join up all the pulmonary veins and then trim the left atrial cuff so it roughly corresponds to that of the recipient. The mitral valve can be inspected and any patent foramen ovale (found in $20-25 \%$ of donor hearts) can be closed. The great vessels are then trimmed to an appropriate length and the aortic and pulmonary valves examined.

\section{Implantation}

With the heart still out of the chest the first suture is placed on the left atrium, starting at the site of the left superior pulmonary. After a couple of stitches have been placed, the heart is put in the chest, pulled to the left by the assistant's hand and the suture tightened. The line runs inferiorly, and then across to the right, terminating adjacent to the IVC orifice. The other end of the first suture is then run across the roof of the atrium, and downwards to join the first suture. There is an option to place a vent into the left atrium at this stage.

The orientation is determined by this first left atrial suture. If it is correct, the other structures-pulmonary artery and aorta, and the two cavae, lie adjacent to each other, and can simply be anastomosed end to end with running sutures. Length must be carefully judged; both redundant tissue, which leads to kinking, and overtrimming, with tension on suture lines, must be avoided.

The so-called "warm" or implantation time can be reduced by only performing the left atrial and great vessel anastomoses before removing the cross-clamp and re- perfusing. Some surgeons will only do the left atrial and aortic anastomoses, doing all the right-sided suture lines with the heart re-perfused and beating.

\section{Reperfusion and completion}

Regardless of the approach, the heart is reperfused after removal of the cross-clamp. De-airing is as for any extensive intra-cardiac procedure and care taken to avoid distension of either ventricle. It is routine to have reperfusion period of at least 30 minutes, whilst the patient is rewarmed. Both atrial and ventricular pacemaker wires are placed and exteriorised. The heart is slowly weaned off cardiopulmonary bypass, taking particular care with right ventricular performance. A temporary left atrial monitoring line is very useful at this stage.

Once off bypass, two mediastinal drains are placed and protamine administered. The chest is closed in the standard fashion.

\section{Comments}

A range of manoeuvres can be adopted to optimise myocardial protection during implantation. Topical cooling of the heart, with exchange of cold saline in the pericardial cavity, is commonly used. We routinely run ice-cold Ringer's solution into the left atrium via the vent. This both cools the inside of the left heart as well as facilitating de-airing. Others run continuous retrograde cold, blood cardioplegia into the coronary sinus during implantation. Another option is to give antegrade cardioplegia via an aortic cannula at regular intervals during implantation.

\section{Clinical results}

This bicaval technique has been used in Newcastle for over 20 years, since 1995, in 809 cardiac transplants, 290 of them in children. In this cohort, the 30 -day survival is $88 \%$, and 20 -year survival $34 \%$ with a median survival of 14 years.

\section{Advantages}

The video shows a very expeditious implantation performed by Dr. Sergey Boldyrev and his team. The excision is entirely standard. In this case, the whole implantation was performed before reperfusion, which allows a clear view of the suture lines and rapid sequence of anastomoses. The ease of this technique is well shown, as is the very good early function, with no bleeding from the suture lines, sinus 
rhythm without pacing and excellent cardiac function.

\section{Acknowledgements}

The research was funded by the National Institute for Health Research Blood and Transplant Research Unit (NIHR BTRU) in Organ Donation and Transplantation at the University of Cambridge in collaboration with Newcastle University and in partnership with NHS Blood and Transplant (NHSBT). The views expressed are those of the author(s) and not necessarily those of the NHS, the NIHR, the Department of Health or NHSBT.

\section{Footnote}

Conflicts of Interest: The authors have no conflicts of interest

Cite this article as: Jungschleger JG, Boldyrev SY, Kaleda VI, Dark JH. Standard orthotopic heart transplantation. Ann Cardiothorac Surg 2018;7(1):169-171. doi: 10.21037/ acs.2018.01.18 to declare.

\section{References}

1. Lower RR, Shumway NE. Studies on orthotopic homotransplantations of the canine heart. Surg Forum 1960;11:18-9.

2. Sievers HH, Weyand M, Kraatz EG, et al. An alternative technique for orthotopic cardiac transplantation with preservation of the normal anatomy of the right atrium. Thorac Cardiovasc Surg 1991;39:70-2.

3. Dreyfus G, Jebara V, Mihaileanu S, et al. Total orthotopic heart transplantation: an alternative to the standard technique. Ann Thorac Surg 1991;52:1181-4. 